\title{
Médiévales
}

Langues, Textes, Histoire

59 | automne 2010

Théâtres du Moyen Âge

\section{De l'oral à l'oral : réflexions sur la transmission écrite des textes dramatiques au Moyen Âge}

From the oral to the oral: reflections on the written transmission of dramatic

texts in the Middle Ages

Taku Kuroiwa, Xavier Leroux et Darwin Smith

\section{CpenEdition}

\section{Journals}

Édition électronique

URL : https://journals.openedition.org/medievales/6056

DOI : 10.4000/medievales.6056

ISSN : $1777-5892$

Éditeur

Presses universitaires de Vincennes

Édition imprimée

Date de publication : 31 décembre 2010

Pagination : 17-40

ISBN : 978-2-84292-267-2

ISSN : 0751-2708

\section{Référence électronique}

Taku Kuroiwa, Xavier Leroux et Darwin Smith, « De l'oral à l'oral : réflexions sur la transmission écrite des textes dramatiques au Moyen Âge », Médiévales [En ligne], 59 | automne 2010, mis en ligne le 20 mars 2013, consulté le 22 avril 2022. URL : http://journals.openedition.org/medievales/6056 ; DOI : https://doi.org/10.4000/medievales.6056 
Médiévales 59, automne 2010, p. 17-39

Taku KUROIWA

Xavier LEROUX

Darwin SMITH

\section{DE L'ORAL À L'ORAL: \\ RÉFLEXIONS SUR LA TRANSMISSION ÉCRITE DES TEXTES DRAMATIQUES AU MOYEN ÂGE}

En France du moins, l'histoire du théâtre médiéval, compris au sens large (autrement dit jusqu'à la première moitié $\mathrm{du} \mathrm{XVI}^{\mathrm{e}}$ siècle), naît sur un quiproquo, une méprise dont les effets marquent encore souvent la critique moderne. Un quiproquo consiste à prendre une chose pour une autre ou pour ce qu'elle n'est pas. En l'occurrence, les textes manuscrits ou imprimés des œuvres dramatiques du Moyen Âge ont été confondus avec les textes dits lors d'une performance collective ou individualisée. Depuis la période classique et pour la grande majorité des œuvres de théâtre, la fixation presque définitive du texte par l'écrit précède son passage à la scène ${ }^{1}$. Le texte écrit constitue la référence ou le modèle de chaque oralisation, qui reproduit, à quelques variantes près, le texte publié 2 .

Au Moyen Âge, dans une civilisation essentiellement orale, le passage à l'écrit du texte dramatique ne paraît pas intervenir de la même façon dans le processus complexe qui règle la transmission et la conservation des œuvres de théâtre. Du fait de la méprise expliquée ci-dessus, on a longtemps considéré que l'origine du théâtre médiéval se confondait avec les plus anciens tex-

1. Nous renvoyons à Pierre Larthomas dont le corpus est d'abord composé de pièces classiques et qui écrit: «Nous avons vu que l'écrit y [i.e. au théâtre] précède le dit. "Il (le théâtre) est d'abord un texte", écrit encore Gaëtan Picon» (P. Larthomas, Le Langage dramatique, Paris, 2001 [1 ${ }^{\text {re }}$ éd. : 1972], p. 30).

2. Quand la critique génétique envisage le cas de quelques pièces connues sous différentes versions, la justification de chacune d'elles comme œuvre à part entière reste fondée sur le principe que l'écrit fait autorité, sans tenir compte de la place de l'oral dans l'élaboration du texte (voir un rappel de ces cas dans J.-M. ADAM, «Réécritures et variation: pour une génétique linguistique et textuelle», Modèles linguistiques, 30/59, 2009, p. 23-50, p. 24). 
tes exhumés. Selon une perspective évolutionniste aujourd'hui discutée, le théâtre français serait né au XII ${ }^{\mathrm{e}}$ siècle avec les premiers témoins scriptuaires, pour engendrer ensuite les divers genres sérieux et comiques - miracles, mystères, moralités, farces et sotties - que nous tentons en vain d'orthonormer. $\mathrm{Au} \mathrm{XIII}^{\mathrm{e}}$ siècle, cet héritage se serait traduit par l'écriture de pièces telles que le Jeu d'Adam, le Jeu de saint Nicolas de Jean Bodel, le Miracle de Théophile de Rutebeuf, le Jeu de Robin et Marion d'Adam de la Halle. Mais l'origine du théâtre, intrinsèquement lié à l'oralisation, ne peut qu'avoir été antérieure à ces premiers témoins, dont la facture montre un degré certain d'élaboration.

Il convient donc de reconsidérer la nature et la fonction des témoins médiévaux dans leur ensemble et, par rapport au processus de conservation écrite, de déterminer, en amont, le statut du texte à enregistrer et, en aval, la finalité de cette transcription ${ }^{3}$.

\section{Formatage du discours dramatique}

Étant donné l'ambitus temporel auquel nous confrontent les témoins scriptuaires datés du milieu du XIII ${ }^{\mathrm{e}}$ jusqu'au milieu du XVI ${ }^{\mathrm{e}}$ siècle ${ }^{4}$, il serait

3. L'importance et la diffusion des travaux de l'anthropologue Jack Goody sur le rapport oral/ écrit et sur la théorie de l'interprétation dans les sociétés contemporaines, anciennes et médiévales, nous conduisent à préciser notre position. Pour J. Goody, il n'y a de culture orale que dans les sociétés sans écriture et la versification n'est pas un facteur d'oralité ; il la conteste comme telle, même là où cela semble aller de soi: «bon nombre des procédés que nous croyons oraux, comme les assonances dans Beowulf (ou dans l'œuvre de Gerald Manley Hopkins), la structure mnémonique du Rig-Veda, la composition formulaire des Grecs, et même l'utilisation massive de la rime, semblent rares dans les cultures sans écrit» (J. Goody, Pouvoirs et savoirs de l'écrit, «La mémoire dans la tradition orale», trad. fr. Paris, 2007, p. 52). J. Goody insiste souvent sur les capacités limitées de formalisation mémorielle au sein des cultures orales par rapport aux sociétés de culture écrite, l'écrit étant un facteur clé, qualitatif, dans le développement du savoir et de la connaissance: «Les institutions sociales sont très affectées par les limitations du canal oral [...]. Les procédures légales sont moins gouvernées par des lois générales, par des procédures formelles» (ibid., p. 49). Suivre J. Goody dans ses propositions effacerait d'emblée le sens de notre recherche, dont le propos est ailleurs: mettre à jour la technicité de procédés de formatage et de formalisation précisément liés au rapport oral / écrit, pour éclairer les pratiques de jeu et d'écriture relatives aux faits dramatiques, enfouies dans le silence des sources. Dans son article «Théâtre, rites et représentations de l'autre» (dans J. Goody, La Peur des représentations, trad. fr. Paris, 2006, p. 113-167), J. Goody expose une vision téléologique du théâtre, des origines à nos jours, où le théâtre médiéval anglais et français est connu de seconde main et selon des stéréotypes anciens: drame religieux né de l'Église et pièces de clercs à caractère urbain, le tout soumis aux interdits de l'Église. En revanche, nous le suivrons dans sa thèse centrale sur le bouleversement que constitue l'apparition de l'écrit dans les processus de communication.

4. Le plus ancien texte identifié comme dramatique est le Jeu d'Adam, conservé dans un manuscrit du milieu du XIII ${ }^{\mathrm{e}}$ siècle (Tours, BM 927). À l'autre extrémité de la période, nous avons les deux manuscrits de la Passion de Valenciennes exécutés trente ans après la représentation de 1547 (Paris, BnF, ms. fr. 12536, et BnF, ms. Rothschild I.7.3). 
péremptoire de généraliser s'agissant des modes de production des œuvres dramatiques. Nous pouvons néanmoins affirmer que celles-ci, pendant toute cette période, sont versifiées. À ce principe, il n'y a pas de contre-exemple, même si, dans le courant du Moyen Âge, on voit évoluer les manifestations de la versification en même temps que son rôle, dans une mesure encore à préciser. Ce changement est fortement marqué par l'apparition en Occident d'un nouveau support d'écriture, le papier, puis par la généralisation massive de son usage au $\mathrm{Xv}^{\mathrm{e}}$ siècle, concomitant de la diffusion des pratiques de l'écrit au-delà des cercles réservés des clercs et des copistes professionnels.

La versification du discours dramatique codifie son enregistrement: sa mise en vers le structure et apparaît comme un facteur actif de sa mémorisation et de sa conservation. À travers cette versification, nous assistons au formatage du discours, autrement dit à son ajustement à un format véhiculaire, en l'occurrence à un système rythmique duplicable à volonté, l'enchaînement d'octosyllabes à rimes plates, sur lequel peuvent se greffer des formes métriques ou strophiques plus élaborées ${ }^{5}$. Ce formatage par la versification n'est cependant pas appliqué au seul discours dramatique : d'autres formes - romanesques, poétiques, juridiques, scientifiques, homilétiques, etc. - ont subi un même processus. Ainsi, dans la première partie de la période médiévale, l'emploi de la versification contribue à la structuration et à la mémorisation d'un vaste corpus. La mise en vers semble une condition (et peut-être l'indice) de l'existence d'une conservation strictement mémorielle d'un texte.

L'expansion progressive et massive de l'écrit modifie les pratiques antérieures en matière d'enregistrement et de lecture des différentes formes de discours. Cette évolution se traduit notamment par le développement d'une abondante littérature en prose au détriment d'une littérature en vers. Avec l'écrit, la conservation du texte est garantie par la pérennité du support matériel. Si la versification favorise encore la mémorisation, elle ne vise plus à assurer la conservation du discours et devient une forme stylisée du texte écrit, un choix de composition.

La forme dramatique est cependant la seule qui échappe totalement à cette évolution: jusqu'au $X I^{\mathrm{e}}$ siècle, nous l'avons déjà dit, elle continue d'être versifiée. À notre connaissance, le fait que la versification n'est systématiquement maintenue que dans ce type de discours n'a jamais fait l'objet d'un commentaire particulier. Nous sommes pourtant confrontés à un phénomène exceptionnel. Étant donné la fonction mémorielle et conservatrice de la versification, son maintien peut sans doute s'expliquer par le constant vaet-vient, consubstantiel à l'activité dramatique au Moyen Âge, entre l'enre-

5. Pour replacer ce phénomène dans une perspective plus large, soulignons que ce type de formatage peut être doublé d'un formatage musical. 
gistrement du discours et son activation orale. Il faut donc faire l'hypothèse que, dans la seconde partie du Moyen Âge et jusqu'à l'émergence de la prose au théâtre ${ }^{6}$, le maintien de la versification est directement lié aux spécificités performancielles du théâtre ${ }^{7}$.

Étroitement liée à l'oralité du discours, la versification l'est également à la pratique du jeu. L'examen approfondi de la tradition textuelle de Pathelin montre que la performance de cette œuvre résultait de l'apprentissage par les joueurs d'un texte mémorisé et écrit mentalement (ou textus), une trame de couples d'octosyllabes à rimes plates, déroulée suivant un canevas. Pour des raisons liées à la mémorisation, la matière de plusieurs répliques pouvait être assemblée dans un même segment de textus. Mais, en situation de jeu, les acteurs redistribuaient le textus selon la nécessité des enchaînements. Ainsi, pour passer d'une réplique à la suivante, les joueurs se partageaient généralement les deux vers d'un couple d'octosyllabes à rime plate ${ }^{8}$. En cas de stichomythie, ils séquençaient le textus en fragments selon les circonstances d'une représentation. L'extrait du manuscrit Bigot fourni ci-dessous donne un des rares fragments repérables de textus dont la ponctuation originelle transcrit le séquençage intégré au formatage ${ }^{9}$ :

\author{
Le DrapPIER \\ C'est un tresbon drap de rouen \\ je vous promet et bien drappé \\ PATHELIN \\ Or vrayment j'en suis attrapé \\ car ne n'avoye intencion \\ d'avoir drap • par la mansion \\ de nostre seigneur quant je vins \\ j'avoye mis a part quatre vingz \\ escus pour retraire une rente \\ més vous en arez vingt ou trente \\ car j'en voy trop bien $\bullet$ la couleur \\ m'en plaist trestant que c'est douleur
}

6. Comme on l'a vu dans les autres domaines, l'apparition de la prose au théâtre limitera l'emploi de la versification à une stylisation du discours dramatique.

7. Le lien que la versification entretient avec l'oralité du discours dramatique doit être souligné, car il semble aujourd'hui paradoxal. En effet, dans une dialectique relativement sommaire qui oppose langage parlé et langage écrit, l'emploi de la forme versifiée au théâtre parait artificiel. Ainsi, notre compréhension des pièces médiévales et de la place qu'y occupe la versification risque d'être faussée si elle est sous-tendue par cette conception moderne du discours dramatique.

8. Ce procédé est désigné, depuis le $\mathrm{XIX}^{\mathrm{e}}$ siècle, sous le nom de rime mnémonique.

9. Pour le texte du manuscrit Bigot, l'analyse de la tradition textuelle de Pathelin et la mise en évidence du textus, voir D. Sмiтh, Maistre Pierre Pathelin. Le Miroir d'Orgueil. Texte d'un recueil inédit du $\mathrm{XV}^{e}$ siècle (mss Paris, B.N.F. fr. 1707 \& 15080). Introduction, Édition, Traduction et Notes, Saint-Benoît-du-Sault, 2002. 


\author{
Le DRAPPIER \\ Voire $\bullet$ d'escus $\bullet$ se peult il faire \\ que ceulx dont vous voulez retraire \\ celle rente $\bullet$ prinssent monnoye
}

On constate, d'une part, l'absence d'enchaînement cohérent entre la deuxième réplique du Drapier et celle de Pathelin et, d'autre part, d'une manière très atypique, l'absence de liaison par la rime entre ces deux répliques dont la première s'achève et l'autre commence par un couple d'octosyllabes à rime plate. Aucune lecture de ce passage selon son organisation manuscrite n'est satisfaisante. Il nous faut tenir compte de la ponctuation originale et de la logique du dialogue. En fait, les punctus marquent ici la fragmentation de ces deux répliques en différents éléments qui s'insèrent les uns dans les autres. Ils ont de plus une valeur performancielle, car ils sont placés autour d'un mot dont ils signalent la répétition $^{10}$. Aussi suggérons-nous que l'exclamation «Voire $\bullet »$ du Drapier répond à la première partie de la réplique de Pathelin et que « $\bullet$ d'escus $\bullet$ » répond à la deuxième partie. Une réalisation dramatique possible de cet échange serait la suivante: «- Or vrayment, j’en suis attrapé, car ne n'avoye intencion d'avoir drap. - Voire ? - Voire! - Par la mansion de nostre Seigneur, quant je vins, j' avoye mis à part quatre vings escus pour retraire escus une rente més vous en avez vingt ou trente car j'en voy trop bien... - D'escus? - D'escus ! - Se peut il faire que ceulx dont vous voulez retraire celle rente... ${ }^{11}{ }$

Cet exemple montre clairement le rôle du formatage entre oralité et écriture, ainsi que l'écart entre texte dit et texte mémorisé. Selon nous, ce sont les mêmes procédés dont témoignent déjà, deux siècles auparavant, les variantes textuelles ainsi que les interpolations de rôles et de répliques du Jeu de Robin et Marion. Les traditions textuelles de ces deux ouvres attestent ainsi de l'ancienneté du jeu par textus mémorisé avec extensions interpolées ${ }^{12}$, et donc de l'existence d'un jeu « de métier», durant deux siècles au moins, bien avant qu'il ne se déclare comme tel, en Italie, sous le nom de Commedia dell'Arte ${ }^{13}$.

10. Pour l'analyse des utilisations du punctus, voir D. Sмiтh, Maistre Pierre Pathelin..., op. cit., p. 125-133, n. 97, et, en particulier, p. 130.

11. Voir une autre réalisation possible du même passage dans D. Sмітн, La Farce de Maître Pathelin, Paris, 2008, p. 27 sq.

12. Voir infra «Les interpolations».

13. Le caractère professionnel du jeu est attesté par l'examen de la tradition textuelle de Pathelin, près d'un siècle avant les documents de la Commedia dell'Arte, et est confirmé par les pratiques associatives, avérées par les premiers contrats écrits de joueurs qui apparaissent à la même époque en France (voir l'édition du contrat de 1486 découvert par Élisabeth Lalou, dans D. Sмітн, Maistre Pierre Pathelin..., op. cit., p. 152, n. 152). Sur les contrats d'acteurs, voir aussi dans ce même dossier l'article de M. Bouhaïk-Gironès, «Comment faire l'histoire de l'acteur au Moyen Âge». 
La spécificité de ces pratiques est révélée par l'écart important qu'il nous faut déceler entre le texte écrit, conservé dans de nombreux codices, et le texte dit, définitivement inaccessible. En effet, nous pouvons affirmer que le format rythmique imposé au discours dramatique en vue de sa conservation n'est pas systématiquement reproduit à l'identique lors de son oralisation théâtrale ou non-théâtrale. Contrairement au texte écrit, le texte performé se caractérise par une grande variabilité. Celle-ci se réalise notamment par des interpolations textuelles, par des interversions de personnages pour un même rôle, par la retranscription mouvante de formes telles que le rondeau. Comme nous le verrons plus loin, cette variabilité n'est pas seulement attestée par l'existence de versions divergentes d'un même texte: elle est encore signalée par les irrégularités relevées en plus grand nombre dans les œuvres dramatiques que dans les autres genres de textes ${ }^{14}$. Le discours dramatique est donc soumis à deux tensions contradictoires: d'une part, le formatage qui cherche à ajuster le texte au cadre versifié afin d'en assurer la transmission et la conservation, d'autre part, l'oralisation qui, sans faire éclater le cadre versifié, intervient sur la matière textuelle au gré des réalisations performancielles.

L'écart du texte formaté au texte joué se réalise donc dans la matière textuelle, tant au niveau de sa versification que de son agencement. Il peut encore être accentué par la présence sur scène de personnages dont les répliques ne sont pas toujours données à l'avance (comme celles des diables et des fous, car elles étaient improvisées ou adaptées aux circonstances de la représentation) ou encore par les modalités d'oralisation (chant, cri, hurlement, etc.). Cette mouvance textuelle, qui témoigne pourtant directement de l'expérience performancielle, est encore largement ignorée par la critique. C'est dire les conséquences de cette lacune dans la tradition historiographique. Notre compréhension d'un tel phénomène doit encore être affinée par l'analyse du processus de formalisation que subit le texte dramatique lors de son passage à l'écrit.

\section{Formalisation du texte dramatique}

Alors que le formatage désigne l'enregistrement mental du discours en l'adaptant à un format textuel (en l'occurrence la versification), la formalisation désigne l'enregistrement écrit du texte dramatique en fonction de contraintes liées, d'une part, aux nécessités pratiques du jeu et, d'autre part, à l'anticipation d'éventuelles oralisations, autrement dit de performances collectives ou individualisées, mais hors du cycle performanciel du jeu. Ces contraintes étant sou-

14. Voir infra «Les irrégularités ». 

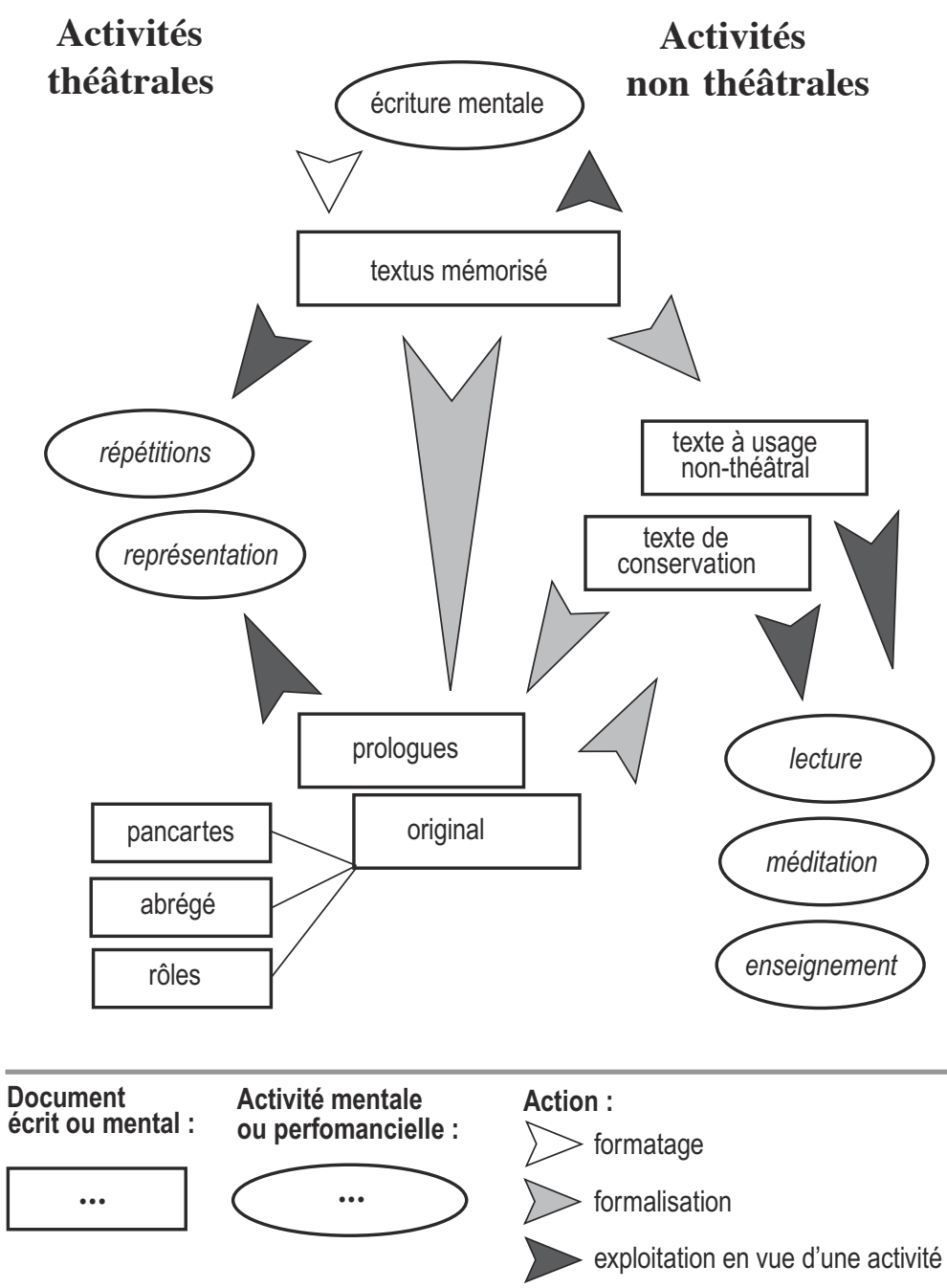

mises à de grandes variations, la formalisation du texte dramatique se réalise de diverses façons selon la période considérée.

L'expansion de l'écrit et des pratiques qui y sont liées transforme les conditions de formalisation de l'œuvre dramatique. Dans les témoins antérieurs $\mathrm{au} \mathrm{Xv}^{\mathrm{e}}$ siècle, systématiquement intégrés à des recueils, la formalisation du texte 
est encore aléatoire ${ }^{15}$, puisqu'on observe, par exemple, que le placement des noms de rôles par rapport aux répliques varie dans chacun des trois manuscrits du jeu de Robin et Marion ${ }^{16}$. À partir de la deuxième moitié du $\mathrm{XV}^{\mathrm{e}}$ siècle, on constate une multiplication massive des manuscrits dits «de théâtre». Les textes ne sont plus systématiquement conservés dans des recueils : ils acquièrent une autonomie livresque qui correspond à l'évolution de leur format. De plus en plus, il s'agit d'œuvres monumentales ${ }^{17}$, dont la performance nécessitait un financement, une organisation collective et une autorité à l'échelon urbain, princier ou royal. Ces dispositifs de performance et la formalisation des textes prenaient place à côté de la tradition du jeu d'acteurs associés, qui se transmettaient leurs pratiques et leurs textus sur canevas et prêtaient leur concours aux représentations urbaines. C'est pour ces jeux, plus généralement appelés «mystères » depuis le XIX ${ }^{\mathrm{e}}$ siècle, que l'on observe le mieux la formalisation de l'écrit dans le cycle du texte dramatique.

\section{Formalisations dans le cycle performanciel}

Au plus près du jeu et de ses répétitions, qui constituent le cycle performanciel du jeu (voir schéma), sont produits les originaux, pancartes, abrégés, rôles et livres des prologues. Ces documents supposent un haut degré de formalisation. L'original, texte de référence pour une représentation dans un temps et un lieu donnés ${ }^{18}$, peut avoir été produit à la table et revêtir les caractéristiques d'un manuscrit d'auteur ${ }^{19}$, ou bien avoir été repris d'une perfor-

15. La question des textes dramatiques les plus anciens en France a été abordée de façon renouvelée par Carol Symes (voir «Early play script: Forms and Functions», Speculum, 77, 2002, p. 778-831), mais du point de vue codicologique, sans tenir compte des phénomènes internes inhérents à ces textes. L'auteur a ultérieurement contextualisé son corpus dans une autre perspective (voir A Common Stage. Theater and Public Life in Medieval Arras, Ithaca, 2007, et le compte rendu de M. Bouhaïk-Gironès, Revue critique de philologie romane, 9, 2008, p. 148157, avec réponse de l'auteur, p. 158-161).

16. Un cas très particulier est constitué par le manuscrit d'Aix de Robin et Marion, qui a enregistré le texte, la musique et l'image, unique témoin d'un système média polyfonctionnel dont l'analyse reste complexe (voir M. CRuse, G. Parussa et I. Ragnard, «The Aix Jeu de Robin et Marion», Studies in Iconography, 25, 2004, p. 1-46).

17. On possède les indications de représentations en plusieurs journées de pièces monumentales pour la première moitié $\mathrm{du} \mathrm{XV}^{\mathrm{e}}$ siècle, mais aucun manuscrit de cette époque ne nous est parvenu.

18. Pour les caractéristiques et fonctions des originaux, livres des prologues et abrégés, voir D. Sмітн, «Les manuscrits de théâtre: introduction codicologique à des manuscrits qui n'existent pas », Gazette du livre médiéval, 33, 1998, p. 1-8.

19. C'est le cas des cahiers du premier jour du Jeu saint Lö̈s (voir D. Sмгтн, Édition critique du jeu saint Loüs, ms. Paris, B.N., fr. 24331, Thèse, université de Paris III, 1987, t. 1, p. 12-23). 
mance précédente ${ }^{20}$, donnant ainsi naissance à ce qui est considéré, in situ, comme un nouvel original ${ }^{21}$. Au cours de la préparation d'une représentation, il peut être produit un deuxième original - ou une deuxième série d'originaux (originalia), puisque le terme est généralement utilisé au pluriel pour désigner l'ensemble des livres ou registres des différentes parties du jeu qui intègre les modifications et corrections des commissaires ou censeurs qui contrôlent l'écriture ou le remaniement du texte, en même temps qu'il est répété par les acteurs ${ }^{22}$.

À partir de l'original, le copiste sélectionne les parties exclusivement nécessaires à l'emploi du matériel qu'il fabrique: les pancartes résultent de l'extraction des indications scéniques géographiques ${ }^{23}$, ou de l'extension performancielle de signes diacritiques présents dans son modèle ${ }^{24}$.

Un abrégé réduit chaque rôle à son premier et dernier vers, en même temps qu'il développe des notes de mise en scène dans une disposition qui permet au régisseur d'avoir simultanément sous les yeux, chaque page étant organisée en deux moitiés égales, d'un côté la «trame orale» (enchaînement des répliques, nombre de vers, noms de rôles et d'acteurs), de l'autre la «trame

20. Voir le cas du manuscrit du Mystère la Passion de Troyes (Troyes, BM 2282), qui cumule les modifications de deux ou trois représentations successives (édité par J.-Cl. BiboLEt, 2 vol., Paris-Genève, Droz, 1987). Mais, faute d'une étude discriminante des différentes mains et de la distribution de leur travail dans la révision du texte, l'éditeur n'en a pas distingué les différentes strates (voir son Introduction, p. XII-XXXII).

21. Voir le cas de la Passion jouée par la ville de Mons, qui emprunte les originaux d'Amiens pour produire ses propres originaux. Sur ces manuscrits, voir également G.A. RunNALls, «La Passion de Mons : étude sur le texte et ses rapports avec la Passion d'Amiens », Revue belge de philologie et d'histoire, 80, 2002, p. 1143-1188.

22. Voir le Mystère des Trois Doms joué à Romans en 1509, qui a fait l'objet d'un premier jeu d'originaux, établi entre août et novembre 1508 à partir du manuscrit de l'auteur, déjà retouché par les commissaires. Fin février 1509, les mêmes ont vaqué plusieurs jours et plusieurs nuits à corriger ces originaux, puis ont fait produire début mars une deuxième série de rôles pour intégrer leurs corrections aux répétitions; un deuxième jeu d'originaux est réalisé au mois d'avril. La représentation a lieu fin mai.

23. Les pancartes (ou briefvets) ne sont mentionnées que dans le compte de la Passion de Mons (Le Livre de conduite du régisseur et le compte des dépenses pour le Mystère de la Passion joué à Mons en 1501, G. CoHen éd., Paris, 1925), mais elles remplissent à l'évidence dans tous les mystères la même fonction de permuter l'affectation nominale des décors, qui peut changer chaque jour ou en cours de journée. Elles peuvent encore être utilisées à d'autres fins. Dans l'abrégé de Mons, une didascalie indique à propos d'Adam: «Il luy donne le nom de Eve» (ibid., p. 12, voir le fac-similé après la p. xx), c'est-à-dire qu'il lui donne une pancarte où est écrit son nom; ce don du panonceau démontre l'origine de l'écriture, au Paradis terrestre, donnée par le premier homme à sa compagne.

24. Sur le crochet, constitutif des originaux, marqueur d'une extension performancielle et dont l'inscription relève du principe du commentaire ou de la glose au texte maître, voir D. SмITH, «Les manuscrits de théâtre...», loc. cit., p. 5. 
logistique » (soit tout le nécessaire au bon déroulement du jeu) ${ }^{25}$. Les rôles rooles, rolets ou parchons - sélectionnent les répliques respectives de chaque personnage ${ }^{26}$. Les livres des prologues enregistrent exclusivement les prologues et épilogues de chaque journée ${ }^{27}$.

Avec ces différents documents, nous sommes confrontés à des procédures de formalisations sélectives dont le support exclusif est le papier, sur une colonne (à pleine page) sans autre justification que par pliage ${ }^{28}$, sans linéation, rubrication ni illustration. Les originaux, abrégés et livres des prologues se signalent enfin par la présence de signes diacritiques, parfois en très grand nombre (plusieurs centaines pour un même original) et par un texte très rarement fautif, puisque copié dans un milieu et des circonstances où il est intelligible pour ceux qui le manient, le transmettent, le jouent. Il faut cependant conclure ce bref aperçu en précisant que les abrégés, pancartes, rôles et autres registres de la pratique, ont presque tous disparu, comme la quasi-totalité des documents de métiers à caractère organique de la même époque - tels qu'affiches, billets d'advertance, livres de feintes (ou de secrets) pour les pratiques dramatiques ${ }^{29}$.

25. L'abrégé de Mons (1501), seul document de régie connu pour cette époque dans le domaine français, est d'une telle perfection pragmatique qu'il ne peut qu'être issu d'une tradition longue, sinon bien établie (voir D. SмiтH, «Un manuscrit de régie médiévale », dans A.-M. Christin dir., Histoire de l'Écriture. De l'idéogramme au multimédia, Paris, 2001, p. 308-309).

26. Sur les rôles, voir É. LALou, «Les rôlets de théâtre, étude codicologique», Actes du $115^{\circ}$ Congrès National des Sociétés Savantes. Théâtre et Spectacles Hier et Aujourd'hui : Moyen Âge et Renaissance [Avignon, 1990], Paris, 1991, p. 51-71.

27. Là encore, le seul livre des prologues parvenu jusqu'à nous est celui de Mons, mais l'existence généralisée de ce dispositif écrit, qui répond à une différenciation organique de la prise de parole dans la performance - une même personne assume la récitation de l'ensemble de prologues et épilogues pour toutes les parties d'un jeu -, explique, d'une part, l'absence de prologues dans les originaux, d'autre part, quand ils sont recopiés, leur disposition irrégulière, tantôt regroupés en tête de copie, tantôt placés au bon endroit du texte dans les exemplaires 'de conservation' exécutés à partir des matériaux écrits du cycle performanciel (voir D. SмIтH, «La question du Prologue de la Passion ou le rôle des formes métriques dans la Creacion du Monde d'Arnoul Gréban », dans L'Économie du dialogue dans l'ancien théâtre européen, textes réunis par J.-P. BoRDIER, Paris, 1999, p. 145 sq.).

28. Sur les pliages particuliers aux originaux et aux abrégés, voir D. SмiтH, «Les manuscrits de théâtre...»,loc. cit., et ID., «Plaidoyer pour l'étude des plis», Gazette du livre médiéval, 42, 2003, p. 1-15: originaux, livres des prologues ou abrégés ont une page divisée en deux parties, mais avec un double pliage inversé pour les originaux et un simple pliage central pour l'abrégé.

29. Affiches annonçant le spectacle (Le Livre de conduite..., op. cit., p. LXVIII-LXIX), billets d'advertance destinés aux machinistes (ibid., p. xc) et livres de feintes (ou de secrets) dont l'unique témoin a été édité par A. Vitale Brovarone, Il quaderno di segreti d'un regista provenzale del Medioevo: Note per la messa in scena d'una Passione, Alessandria, 1984. Comme pour toutes les sociétés d'économie de pénurie, au Moyen Âge comme dans l'Antiquité, ce qui n'avait pas ou plus d'usage envisagé était recyclé, le papier ou le parchemin comme le métal ou la pierre. Ceci expli- 


\section{Formalisations pour des activités non théâtrales}

À partir du matériel établi pour le cycle du jeu sont produites des copies liées à d'autres finalités précises - que nous désignons sur notre schéma sous le nom de «textes de conservation». Comme pour tous les livres manuscrits au Moyen Âge, les usages de destination sont connus de leur exécutant qui sait pour qui et dans quelles conditions sa copie sera utilisée. Très généralement, la formalisation opère alors de façon globalisante et récapitulative, à l'inverse des procédures décrites précédemment. Il y aura, par exemple, intégration physique de ce qui appartenait, dans les originaux, à des groupes de cahiers distincts pour chaque partie de représentation ${ }^{30}$, élimination de tout ou partie du paratexte didascalique, incorporation ou non des prologues et des épilogues, disparition ou transformation des signes diacritiques en pieds de mouche et rubrications, ajout éventuel d'une trame d'éléments visuels. Le texte lui-même peut être soumis à des modifications liées au destinataire individuel ou collectif, aux circonstances pratiques ou linguistiques de la nouvelle utilisation envisagée: ainsi en est-il de la formalisation du texte par dérégionalisation de la langue, comme pour la copie de Robin et Marion du manuscrit d'Aix-en-Provence, dont l'essentiel des picardismes a disparu ${ }^{31}$.

L'interprétation coordonnée de tous les signes de formalisation du témoin permet de repérer des usages individualisés ou collectifs : lecture pieuse, méditation $^{32}$ et enseignement. Ces usages peuvent interagir en fonction des pratiques individuelles. Ainsi, le frère Aubert, de la Chartreuse de Vauvert, près de Paris, copie la Passion d'Arnoul Gréban (Paris, BnF, fr. 15064-15065) au format d'un petit livre d'heures, sur des cahiers in-quarto de papier encartés dans un folio de parchemin, dont le texte comporte des notes à caractère exégético-historique ${ }^{33}$.

que la disparition massive de ce type de matériel et c'est pour la même raison que des fragments de rôles et d'originaux ont régulièrement été retrouvés en bourrage dans des plats de reliures confectionnés à la fin du $\mathrm{XV}^{\mathrm{e}} \mathrm{ou}$ au début du $\mathrm{XVI}^{\mathrm{e}}$ siècle.

30. Les originaux peuvent être divisés en journée ou demi-journées (matinee et aprés disner).

31. Texte édité par G. Parussa dans J.-P. BordiER dir., Théâtre du Moyen Âge, Paris, à paraître. Cette dérégionalisation peut toucher tous types de formalisation, puisque l'original de Mons, copié sur celui d'Amiens, est lui-même constellé de formes wallonnes.

32. Deux bons exemples de livres de méditation à partir de texte «de théâtre» sont les mss. A de la Passion d'Arnoul Gréban (Paris, BnF, fr. 816), dont les didascalies ont été omises (souvent transformées en ponctuation) et quelques notes marginales ajoutées pour articuler la copie à l'argument théologique du texte (voir D. Sмітн, «La question du Prologue de la Passion... », loc. cit., p. 146 sq.), et le Recueil Bigot (composé à l'origine des actuels manuscrits Paris, Bnf, fr. 1707 et 15080), où Maistre Pathelin est articulé à une lecture-démonstration des péchés capitaux, l'Orgueil et l'Avarice étant personnifiés par les personnages de l'Avocat et du Drappier (voir D. SMITH, Maistre Pathelin, Le Miroir d'Orgueil, op. cit., p. 51-64).

33. Avec ce témoin, il y a un format et une association de matières subjectives inhabituels, en apparence, pour ce type de texte, mais correspondant à la production de livres d'heures en milieu 
La pratique individuelle de lecture, mais aussi collective à haute voix, conditionne des didascalies à caractère narratif inspirées des romans en prose ${ }^{34}$. Dans d'autres cas, le manuscrit recourt à des pratiques de mise en page plus «livresques», avec le développement d'enluminures et de peintures. Ce dispositif peut incorporer des dimensions performancielles non textualisées du jeu: par exemple, la première intervention d'un nouveau personnage peut être signalée par une miniature ou une lettrine ${ }^{35}$.

À la différence des documents produits dans le cycle performanciel du jeu, ces formalisations sur parchemin ou papier se font souvent à deux colonnes, au détriment de l'espace marginal réservé aux commentaires didascaliques dans les originaux, où la disposition à pleine page permettait un développement presque illimité et adventice des indications scéniques en marge du texte maître. Les indications scéniques conservées sont alors généralement placées entre deux répliques. Ainsi formalisées, elles sont parfois simultanément formatées et paraissent sous la forme d'octosyllabes à rimes plates plus ou moins intégrées à la chaîne rimique du texte ${ }^{36}$. Enfin, rien n'empêche qu'un document de «conservation» soit ultérieurement réintégré dans le cycle performanciel du jeu: le formatage, en amont, rend la trame textuelle exploitable pour une nouvelle représentation. C'est ce qui se passe au $\mathrm{XvI}^{\mathrm{e}}$ siècle, avec les imprimés réutilisés pour des représentations ${ }^{37}$.

Le passage de l'oral à l'écrit intervient aussi de manière particulière pour les farces, moralités et comédies des acteurs associés, qui jouent par textus séquencé comme on l'a vu pour Pathelin. Là, plus qu'ailleurs, l'oralité conditionne la formalisation. Il existe un nombre infime de manuscrits de ces œuvres, ce qui, selon nous, constitue une preuve indirecte supplémentaire du fonctionnement particulier de leur transmission. La très grande majorité de ces textes n'est connue que par des formalisations tardives, à la suite du démantèlement de

conventuel. Frère Aubert a produit par ailleurs des copies de textes à caractère scolaire et peut avoir fait partie de l'environnement théologien d'Arnoul Gréban qui, rappelons-le, a assuré un semestre de cours sur l'Ancien Testament à la faculté de théologie en 1456-1457 et pour qui d'autres manuscrits témoignent, selon nous, d'une diffusion dans ce milieu (voir D. SMITH, «La question du Prologue de la Passion...», loc. cit., p. 164, n. 58).

34. Voir les didascalies de La Vengeance Nostre Seigneur (Arras, BM 697).

35. Voir R.L.A. Clark et P. SheIngorn, «Performative reading: the illustrated manuscripts of Gréban's Mystère de la Passion», European Medieval Drama, 6, 2002, p. 129-154, et dans ce même dossier l'article de Corneliu Dragomirescu.

36. Voir notamment, dans Courtois d'Arras, les v. 91-95, 102, 146-150 (édité par E. FARAL, Paris, 1922, $2^{\mathrm{e}}$ éd. revue).

37. J. Koopmans, «Jeux d'Apôtres virtuels? Un exemplaire du Mystère des Actes des Apôtres annoté du XVI ${ }^{\mathrm{e}}$ siècle», dans European theatre: 1470-1600, traditions and transformations, textes réunis par M. Gosman et R. Walthaus, Groningen, 1996, p. 31-42. 
l'association qui gérait le «capital textuel ${ }^{38}$. Ce qui nous parvient cumule souvent plusieurs strates d'élaboration, de formatage et de formalisation.

\section{Phénomènes de rationalisation}

Dans la formalisation du texte dramatique, sur un plan général, plusieurs exigences sont à l'œuvre, que recouvre la notion de rationalisation. Cette tendance à l'économie vise à réduire à sa plus simple expression tout ce qui, dans le texte, touche au phénomène de répétition: on ne répétera pas nécessairement à l'écrit ce qui doit l'être à l'oral ${ }^{39}$. Cette économie textuelle est le reflet d'une pratique performancielle qui amplifiait par la répétition ce qu'il fallait souligner par la voix en le distribuant à plusieurs reprises dans l'espace scénique ${ }^{40}$.

L'effort de rationalisation porte notamment sur une difficulté inhérente au passage de l'oral à l'écrit, celle de la simultanéité, c'est-à-dire des textes parlés, criés ou chantés en voix simultanées, une situation constitutive du dialogue dramatique. Ce problème est résolu par le rondeau, utilisé comme indicateur conventionnel de simultanéité. Sa présence indique aux lecteurs ou aux joueurs que la disposition du texte n'est que la formalisation de ce qui sera lu, pensé, joué ad libitum ou selon un code d'organisation vocale destiné à reproduire l'enchevêtrement verbal d'une situation collective (repas, salutations, célébrations, mais également combats, séances de tortures, etc.). Au XvI ${ }^{\mathrm{e}}$ siècle, un auteur comme Gérard de Vivre proposera une nouvelle codification, par signes typographiques, pour remplacer de telles modalités formelles de l'inscription versifiée ${ }^{41}$.

38. Sur cette question, voir. D. Sмiтн, Maistre Pierre Pathelin..., op. cit., p. 32-33.

39. À l'écrit, la répétition est reproduite in extenso (à l'identique ou avec des variantes) ou de manière abrégée (notamment par l'emploi de la formule «etc.» pour des vers répétés à l'intérieur de formes fixes); la répétition peut n'être qu'annoncée, par des signes diacritiques (crochets, punctus, etc.) ou une didascalie plus ou moins explicite, comme «ces huit lignes se doivent dire troiz fois» (en marge du monologue de Cassandre dans la Destruction de Troyes, dans plusieurs des témoins de la tradition manuscrite, comme Édimbourg, National Library of Scotland, Adv. MS. 191.9, fo $298 \mathrm{v}^{\circ}$ ), ou par la simple formule «redictes» (D. Sмгтн, Le Jeu saint Lö̈s, op. cit., t. 1, p. 86).

40. Rien de moins évident pour le lecteur contemporain ou pour les spécialistes de théâtre et de littérature du Moyen Âge, qui commencent seulement à s'occuper du phénomène en tant que tel. L'évidence de cette pratique est masquée par la disparition de la répétition expressis verbis, dont on trouve de beaux exemples dans la littérature dramatique médiévale germanique (voir D. SмITH, Maistre Pierre Pathelin..., op. cit., p. 154, n. 156).

41. Voir les tables de signes proposées par Gérard de Vivre à la suite des préfaces à ses éditions de La Comédie des Amours (1577), La Comédie de la Fidélité nuptiale (1578) et plusieurs autres. Il utilise des signes typographiques pour signifier le rythme du discours (parler bas, plus vite, plus lentement) ou l'enchevêtrement de la parole simultanée. 
La rationalisation formalisante concerne aussi les éléments permettant une extension performancielle du texte en fonction d'exigences pratiques potentielles. Ceci explique le caractère ad libitum de nombreuses didascalies aussi bien sur les originaux que sur les «copies de conservation» - «Lez genz du parc trestous, s'on veut, crient Noel, et ilz vont a l'eglise $»^{42}-$, des suggestions alternatives du type: «S'il veut aller à la mule, le secretaire dit ceste ligne: Velacy, sire, en harnaz bel ${ }^{43}$ » ou de la possibilité, à un niveau macrostructural, de composer et décomposer un cycle de pièces dramatiques ${ }^{44}$.

En outre, le document n'enregistre que ce qu'il doit permettre de faire par la lecture ou le jeu, et donc rien de ce qui appartient à d'autres pratiques: c'est pourquoi il n'y a quasiment jamais de musique dans les témoins scriptuaires, même si les traces de sa réalisation vocale ou instrumentale sont nombreuses. Les rares exceptions connues sont celles où la musique est si particulière qu'elle est notée, comme celle des Juifs dans la Passion de Semur ${ }^{45}$.

En définitive, le document écrit répond à une finalité propre dont on ne possède que de rares indices directs ${ }^{46}$. La plupart des témoins échappent à toute espèce de catégorisation. Il est donc essentiel de s'affranchir de toute conception évolutionniste et linéaire de la vie des textes de théâtre ${ }^{47}$. Dans cette perspective, notre proposition vise à éclairer les pratiques d'élaboration du texte, déterminée par les plus ou moins nombreux passages entre l'oral et l'écrit ${ }^{48}$. Le degré de cette élaboration des œuvres ne se vérifie pas seulement dans la régu-

42. D. Sмітн, Le Jeu saint Loÿs..., op. cit., t. 1, après les v. 444.

43. Ibid., après les v. 880 .

44. G. PARUSSA, «Le manuscrit 1131 de la Bibliothèque Sainte-Geneviève de Paris : un montage signifiant?», dans Mouvances et jointures. Du manuscrit au texte médiéval, textes réunis par Milena Mikhaïlova, Orléans, 2005, p. 229-251.

45. The Passion de Semur, P.T. Durbin et L. Muir éds., Leeds, 1981, p. 65-66.

46. Le texte liminaire du ms. A de la Passion d'Arnoul Gréban, qui donne l'argument théologique de l'œuvre et sert de repère à sa formalisation en tant que livre de méditation, est exceptionnel. De même, dans les recueils, est rarissime la présence d'un argument exprimant les raisons internes (et souvent multiples) de l'assemblage. La copie même d'un mystère peut faire coïncider divers intérêts : le spectacle, le jeu, la méditation, l'histoire, etc.

47. D. Smith et É. Lalou sont restés très prudents, dans le premier article consacré à la typologie des manuscrits de théâtre («Pour une typologie des manuscrits de théâtre médiéval», dans $L e$ Théâtre et la cité, Actes du Ve Colloque de la SITM, Fifteenth Century Studies, 13, 1988, p. 569-579). G.A. Runnalls, qui a suivi dans cette voie, avec «Towards a Typology of Medieval French Mystery Play Manuscripts » (dans The Editor and the Text, textes réunis par P.E. BENNETT et G.A. RunNALLs, édimbourg, 1990, p. 96-113), a développé inversement une typologie très normée, avec des modèles qui ne sont pas articulés sur l'établissement de pratiques ni sur des critères internes (textuels), mais seulement sur des observations codicologiques qui ne sont pas rapportées aux pratiques manuscrites en général; G.A. Runnalls admet lui-même que les types qu'il distingue se chevauchent.

48. Les limites de cet article ne nous permettent pas de rendre compte ici de la tradition de la Passion d'Arnoul Gréban, qui fonctionne par remaniements successifs jusqu'au milieu du XVI ${ }^{\mathrm{e}}$ siècle. 
larité de leur versification, mais aussi dans les traces plus ou moins perceptibles de remaniements antérieurs et de leur activité performancielle.

\section{Les témoins de l'élaboration du texte}

\section{Les interpolations}

Les interpolations observées dans les textes dramatiques à témoins multiples, du XIII ${ }^{\mathrm{e}}$ au XVI ${ }^{\mathrm{e}}$ siècle, font apparaître d'étonnantes convergences de modalités techniques pour insérer quelques vers, une réplique ou une scène entière, aux jointures de la trame octosyllabique. Les plus brèves tiennent de l'improvisation réglée en s'appuyant sur la répétition d'interjections, d'adresses ou d'expressions figées exprimant le doute, l'exclamation ou l'interrogation, avec des effets de stichomythie. Les plus longues tiennent de la scène de genre, comme les diableries ou les scènes de «métiers» (marins, bourreaux, maçons, etc.). Pour que l'interpolation fonctionne, elle doit répondre à un impératif interne ou contextuel. Dans la réécriture du discours théâtral, l'interpolation atteste d'une permanence dans la relation du texte à l'espace du jeu, d'une pratique toujours liée à une oralisation future.

Le procédé d'interpolation le plus facilement identifiable est celui de la «quadruple rime chevauchante» ${ }^{49}$. Il consiste à dupliquer la rime d'un couple à rime plate, là où l'on souhaite faire une insertion. On crée ainsi un deuxième couple dont les vers sont disposés aux extrémités du texte interpolé; le tout est ensuite inséré dans la trame du texte entre les deux vers du couple modèle, et les vers de suture fonctionnent comme des charnières. Pour son édition de Robin et Marion (Paris, BnF, fr. 25566, ms. P) ${ }^{50}$, Ernest Langlois, qui a rejeté les interpolations de son manuscrit de base, n'a pas vu - ni d'ailleurs aucun autre éditeur après lui une interpolation supplémentaire, également présente dans le ms. Pa (Paris, BnF, fr. 1569), mais absente du ms. A (Aix, Méjanes 166). C'est le plus ancien exemple que nous connaissions de quadruple rime chevauchante, ici suivi d'une permutation des rôles, chaque version demeurant parfaitement cohérente, indice supplémentaire, s'il en était besoin, de l'origine performancielle de l'interpolation.

49. R. LebÈGue, «Fonction de la quadruple rime mnémonique chevauchante», Comptes rendus de l'Académie des Inscriptions et Belles-Lettres, 1960, p. $92-96$ [repris dans ID., Études sur le théâtre français, t. I, Paris, 1977, p. 24-28]. Ce procédé a été exposé pour la première fois par l'auteur dans Le Mystère des Actes des Apôtres. Contribution à l'histoire du protestantisme français au XVI siècle, Paris, 1929, p. 48-53.

50. E. Langlois s'est longuement justifié de ce choix (E. LANGLoIs, «Interpolations du jeu de Robin et Marion», Romania, 24, 1895, p. 437-446), sur lequel il a fondé ses éditions: Le Jeu de Robin et Marion, Paris, 1896, et Adam le Bossu, trouvère artésien du XIIt siècle. Le Jeu de Robin et Marion, suivi du Jeu du Pèlerin, Paris, 1924. 
MARIONS

Et encore

esgarde comme est reveleus

RoBIN

Diex con je seroie ja preus

Se li chevaliers revenoit

MARIONS

Voirement robin que che doit Que tu ne ses par quel engien Je m'escapai

RoBIN

$$
\text { Je le soi bien }
$$

Nous veïsmes tout ton couvin Demandes baudon men cousin Et gautier quant t'en vi partir S'il orent en moi que tenir trois fois leur escapai tous deus

Interpolation

(éd. Langlois, v. 416-425)

MARIONS

Robin tu ies trop corageus

Mais quant li cose est bien allee

De legier doit estre ouvliee ne nus ne le doit point reprendre

Baudons (P) Marion (Pa) Robin (A)

Il nous couvient huart atendre

et peronnele qui venront

Ou ves les chi

Gautiers (P) Baudons (Pa) Marote (A)

Voirement sont...

Le procédé de quadruple rime chevauchante permet de localiser l'interpolation même dans une tradition connue par un témoin unique. Ainsi en est-il, deux siècles plus tard, dans le Mystère de saint Vincent ${ }^{51}$ :

HANEQuin

Nenny, dea, deux et deux, ung page, beaux gaillars et non pas lais!

Il n'yra que jusqu'au palays parler a Dioclician.

La sera maint sage ancien et maint homme d'entendement. 


\section{GRINGAUT}

Nous partiron presentement, puis qu'il luy plaist, sans contredire. Dictes! Voulez vous plus rien dire? Dictes le nous apertement. Interpolation

Voulentiers et legierement nous le ferons, puis qu'il luy plaist, car le servir ne nous desplaist.

Tout incontinent nous allons.

On remarquera la technicité du procédé qui ne comporte pas seulement la composition d'une double rime pour enchâsser l'interpolation mais inclut, dans les deux cas, une répétition par anticipation d'un fragment du texte de base pour faire aussi une charnière de contenu («voirement», « puis qu'il luy plaist»). D’autres types d'interpolations utilisent la coupe de vers ou la substitution de rime qui rendent l'interpolation difficilement repérable en cas de témoin unique ${ }^{52}$.

\section{Les rondeaux}

Certaines situations-types paraissent plus propices à un formatage sur le modèle de structures versifiées préexistantes, en l'occurrence le rondeau ${ }^{53}$. Dans les textes dramatiques, la forme de rondeau la plus fréquente reste le triolet, sur le schéma $A B a A a b A B$. Toutefois, dans les multiples témoins de la Passion d'Arnoul Gréban, il arrive que les différentes versions d'un même passage supposé former un triolet présentent des configurations métriques et rimiques très éloignées du modèle canonique ${ }^{54}$. L'exemple le plus éloquent est celui du triolet du massacre des Innocents, que nous rapportons d'abord tel qu'il apparaît dans l'édition d'Omer Jodogne ${ }^{55}$ :

52. Voir l'analyse détaillée de ces procédés dans D. Sмiтн, Maistre Pierre Pathelin..., op. cit., p. $82-96$.

53. Le rondeau «s'emploie dans toutes sortes de situations où la nature de l'action requiert, pour quelque raison que ce soit, la répétition de certaines répliques» (W. NoOMEN, Étude sur les formes métriques du Mystère du Vieil Testament, Amsterdam, 1962, p. 96).

54. L'exploitation du schéma modèle du triolet peut être divisée en trois catégories, à savoir, la simplification (qui va parfois jusqu'à la dissolution de la forme dans la trame environnante), le maintien (qui conserve ainsi la stylisation du discours assurée par le modèle d'écriture courant) et l'amplification. Pour l'analyse plus développée des occurrences du triolet dans les manuscrits de la Passion d'Arnoul Gréban, voir T. Kuroiwa, «"Le viel jeu” en mouvement: la configuration rimique et métrique des triolets dans les manuscrits du Mystère de la Passion (la Creacion du Monde et la Première Journée) d'Arnoul Gréban », dans Vers une poétique du discours dramatique au Moyen Âge, Actes du colloque organisé au Palais Neptune de Toulon les 13 et 14 novembre 2008, textes réunis par X. Leroux, Paris, Babeliana 14, 2011, p. 143-157.

55. Le Mystère de la Passion d'Arnoul Gréban, O. Jodogne éd., Bruxelles, t. I (1965), t. II (1983), t. I, p. 106, v. 7714-7721. 
Arbeline

Que feras tu, mauvais tirant?

Laisse le povre innocent vivre.

A

AgripPART

Se je n'ay tost ung secourant...

Arbeline

Que feras tu, mauvais tirant?

AgripPart

Ceste lice est si fort tirant

que j'ay paour qu'elle me delivre.

ANDROMETA

Que feras tu, mauvais tirant?

Laisse le povre innocent vivre.

B

$a$

A

$a$

$b$

A

$B$

Mais, alors que la présence de ce rondeau se justifie pleinement du point de vue scénique ou rhétorique, aucun manuscrit ne reproduit en fait ce triolet sous cette forme. Deux manuscrits seulement permettent d'aboutir à cette version, les vers manquants ayant été ajoutés dans la marge ${ }^{56}$. En entérinant cette «correction», O. Jodogne a lui-même effectué un «reformatage» du texte pour son enregistrement au $\mathrm{xx}^{\mathrm{e}}$ siècle, ainsi qu'un travail de formalisation par la dilatation de l'interlettrage dans les vers-refrains.

Du fait des pratiques de formatage et de formalisation décrites précédemment, cette mouvance textuelle ne devait pas sembler problématique. Les différentes versions conservées dans les manuscrits peuvent résulter de la formalisation spécifique à chaque témoin scriptuaire, voire du formatage initial effectué par l'auteur (ou d'un reformatage établi par un remanieur). En outre, lors d'une activation performancielle, elles peuvent avoir été réalisées de manières textuellement différentes, mais suffisamment convaincantes. Cette pratique est également présumée par la présence de signes diacritiques en marge de certaines occurrences. Nous trouvons un éventuel reflet d'une 'libre' activation orale du même triolet dans la réalisation du manuscrit de Rome (ms. D), où le passage est transformé par l'insertion de vers interpolés, mais bien clos par le second membre du refrain initial ${ }^{57}$ :

\section{Arbeline}

Que feras tu, mauvaiz tirant?

A

Laisse le povre innocent vivre!

AGRIPART

56. L'apparat critique d'O. Jodogne ne correspond pas, selon notre enquête, à la réalité textuelle des manuscrits qu'il a transcrits : tous les exemples, du moins au premier abord, sont dépourvus du refrain $A$ intermédiaire, même dans le manuscrit de base (ms. B); dans les mss $\mathrm{C}$ et $\mathrm{G}$, les vers «absents » sont en fait ajoutés en marge.

57. Ms. D: Rome, Accademia dei Lincei, Corsini 44 A. $7, \mathrm{f}^{\circ} 135 \mathrm{r}^{\circ}-\mathrm{v}^{\circ}$; nous ponctuons en éliminant les ponctuations originales et distinguons, selon 1'usage, $i$ et $j$, ainsi que $u$ et $v$. 
Si je n'ay tost ung secourant,

Cette lisse est se fort tirant

Que j'ay peur qu'il me delivre.

ANDROMETA

Que feras tu, mauvaiz tirant?

Que feras tu, dy, es tu yvre?

Si part tout te devoye suivre,

Se n'aras tu mon filz jamaiz!

Je te promestz,

Sy je m'y metz,

Que tu le lerras sans doubter.

$\mathrm{Ne}$ te entremestz

Plus desormaitz

De me venir tant raboucter!

Helas, vueille moy escouter,

Laysse le povre innocent vivre!

$B$

À l'opposé, la scène correspondante est formalisée par les deux seuls versrefrains $A B$ dans le manuscrit déjà mentionné du frère Aubert, resitués ci-dessous dans leur contexte ${ }^{58}$ :

(ARFAXAT

Despesche en la court, Agrippart,

Et nous en allons tout courant.)

ARbELINE

Que feras tu, mauvais tyrant?

Laisse le povre innocent vivre!

A

(AgRIPPART

$B$

Sçay je bien ma leçon sans livre?...)

Il nous est impossible de déterminer si cette version reflète le texte joué ou plutôt le travail du copiste: le signe «‡» que nous trouvons à gauche dans la marge du manuscrit peut éventuellement appuyer l'hypothèse que celui-ci n'a pas copié le reste du triolet pour des raisons que nous ignorons. À travers ces exemples, il nous est permis de constater la manière dont un modèle d'écriture (ici la «forme fixe» $A B a A a b A B$ ) peut servir d'outil conceptuel lors du formatage et/ou de la formalisation du discours dramatique pour indiquer la répétition. Mais les témoins scriptuaires connus ne poursuivent pas fidèlement le schéma rimique et métrique de ce modèle, mouvance encore accentuée par la liberté qui a pu exister lors de l'activation orale de ce passage.

58. Ms. F: Paris, BnF, fr. $15064, \mathrm{f}^{\circ} 147 \mathrm{v}^{\circ}$; nous ponctuons et distinguons certains phonèmes selon l'usage. 


\section{Les irrégularités}

La présence d'irrégularités dans la versification est fréquemment considérée comme la preuve de l'incurie du scribe et, conjointement, de l'altération du texte conservé par comparaison avec d'autres témoins d'un même texte, quand le témoin scriptuaire s'inscrit dans une tradition textuelle, ou avec un modèle archétypal présupposé, lorsqu'il s'agit d'un isolat.

Nous distinguons deux types d'irrégularités. Le premier porte sur la rime, le second sur le mètre. Les vers sont dits orphelins lorsqu'ils ne riment avec aucun autre, ils sont dits hypermétriques ou hypométriques selon qu'ils comptent une ou plusieurs syllabes de plus ou de moins que ce que laisse présumer leur entourage. Ces deux formes d'irrégularités apparaissent le plus souvent simultanément et l'examen des unes reste lié à celui des autres. Pour juger de l'irrégularité d'un vers, l'analyse syntaxique demeure un critère fiable. Si la syntaxe du passage incriminé est incorrecte, le texte est vraisemblablement fautif, encore que la faute n'ait pas nécessairement été commise par le copiste et puisse être inhérente au modèle. Mais, bien souvent, ces irrégularités n'altèrent pas la syntaxe du texte.

Les efforts de rationalisation décrits précédemment peuvent être à l'origine d'irrégularités, notamment pour ce qui touche aux phénomènes de répétition ou d'omission délibérée. La dérégionalisation de la langue du texte est un autre facteur à prendre en compte. Mais notre méconnaissance des codes de lecture et de diction des textes dramatiques est plus certainement en cause. Or, dans ce domaine, le principe directeur paraît bien être une grande flexibilité dans l'application de règles versificatoires supposées contraignantes, mais à ce jour bien imprécises, étant donné l'absence d'informations fournies par les arts rhétoriques de l'époque en matière théâtrale.

Le cas du Mistere de la tressainte Conception nous paraît exemplaire ${ }^{59}$. Cet unicum se signale par sa très grande irrégularité ${ }^{60}$. De cette profusion d'anomalies se dégagent cependant des lignes directrices qui tendent à régler l'emploi de ces irrégularités qui, dès lors, ne sauraient être encore considérées comme telles. En effet, on s'aperçoit que les passages les plus suspects substituent à la rime une autre forme de répétition. Dans l'extrait suivant, les vers 8069, 8070 et 8071 ne riment avec aucun autre:

59. Texte disqualifié par É. Roy dans Le Mystère de la Passion en France du XIVe et du XVe siècle. Étude sur les sources et le classement des mystères de la Passion, accompagnée de textes iné-

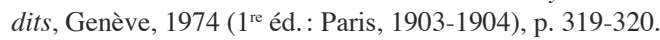

60. Voir X. Leroux, Le Mystère de la Conception (Chantilly, ms. Condé 616), Thèse, université de Paris IV, 2003, t. 1, p. 105-139, et ID., « Essai de localisation du Mistere de la tressainte Conception de la glorieuze Vierge Marie par parsonages (Chantilly, ms. Condé 616)», Revue de Linguistique Romane, 72, 2008, p. 371-412. 
Nostre Dame

Levéz cecy incontinant 8066

et gracez a Dieu je rendrey.

Mon Dieu, je vous louerey. $\quad 8068$

De chesqum soiéz vous loué

et de ces biens cy vous mercie. $\quad 8070$

Graces et mercis je vous rans

des biens que tous jours me fectez. $\quad 8072$

AMPHiBOLIS

Quant noz viandez seront prestez,

Le passage est fondé sur un jeu de répétitions ${ }^{61}$ qui rappelle, du moins dans son principe, la forme du rondeau, dont il a été question plus haut. Or, la situation d'action de grâces, qui caractérise ce passage, est de celles qui favorisent l'emploi du rondeau ${ }^{62}$.

Cette fois encore, on s'aperçoit que se rejoignent, au lieu de s'opposer, des passages a priori irréguliers et des formes versifiées élaborées. La confrontation de ces extrêmes nous invite à reconsidérer dans son ensemble notre compréhension de la versification et de ce qu'il ne convient plus d'appeler systématiquement des 'irrégularités'.

\section{Conclusions}

Nous analysons le processus d'enregistrement du texte dramatique à deux niveaux distincts. Il consiste d'abord, au niveau de sa création et de son élaboration, en la structuration par modulation sonore et rythmique d'un discours syntaxiquement organisé, le formatage. Il procède ensuite d'une notation ou d'une transcription à usage performanciel, la formalisation. Cette réévaluation formelle nous a conduits à repenser la nature du texte versifié, à mettre l'oralité au cœur des enjeux de la transmission écrite de la trame textuelle, à éclairer les irrégularités, insertions et autres procédés d'élaboration comme autant de témoins de la vie de la matière transmise. Dans le domaine spécifique de la versification, nous voyons s'ébaucher un système régi par des principes élémentaires, mais dont le code reste encore à comprendre ${ }^{63}$.

61. Voir: louerey 8068 - loué 8069 ; mercie 8070 - mercis 8071 ; biens 8070, 8072; et gracez a Dieu je rendrey 8067 - graces et mercis je vous rans 8071.

62. Au début de la Passion d'Arnoul Gréban, les anges utilisent un rondeau quatrain pour rendre gloire à Dieu (v. 98-113).

63. Les auteurs du présent article proposent une première hypothèse pour un nouveau paradigme du fonctionnement systémique de la versification dans «Formes fixes: futilités versificatoires ou système de pensée? », dans Vers une poétique..., op. cit., p. 121-142. 
L'examen détaillé des procédés de formatage, de formalisation et d'élaboration du texte n'est pas sans rappeler ce qu'expose Dario Fo, dans Le Gai savoir de l'acteur, sur le colportage des pratiques performancielles chez les héritiers de la Commedia dell'Arte. Au Moyen Âge, le textus mémorisé et séquencé, l'habile facture des interpolations, la réorganisation mouvante du matériau textuel des formes à refrain appartiennent au savoir-faire de l'acteur, transmis de génération en génération, comme en témoignent les traditions manuscrites de Pathelin et Robin et Marion, distantes de plusieurs siècles. Comment imaginer qu'il n'y ait pas eu là des technicités comparables à d'autres pratiques collectives, comme, en musique, celle du cantare super librum (ou technique d'improvisation du «chant sur le livre») qui développait une mélodie en contrepoint à deux ou trois voix selon des règles précises?

Les spécialistes du théâtre médiéval se sont longtemps attachés à distinguer les bons des mauvais manuscrits ${ }^{64}$. Mais des témoins comme le Robin et Marion du manuscrit d'Aix, le Pathelin du recueil Bigot, l'unicum de la Conception ou les variantes de la Passion d'Arnoul Gréban reflètent, sans doute plus que les textes dits «réguliers», les métamorphoses de la mouvance performancielle. Les critères d'analyse que nous avons tenté d'établir permettent de réévaluer les différents représentants d'une famille en termes, non de qualité, mais de nature et de fonction. Appliqués à des isolats, les mêmes critères permettent de resituer ces œuvres au sein des processus d'élaboration du texte dramatique au Moyen Âge. Nous aboutissons ainsi à une conception globalisante des mécanismes de production mentale et écrite qui rejoint les avancées actuelles de la critique génétique, à ceci près que notre analyse intègre l'oralité à la genèse du texte.

On ne dira jamais trop ce que l'écriture médiévale doit aux multiples oralisations réalisées par des groupes d'acteurs, voire des communautés de lecteurs. Les textes qui nous sont parvenus ne sont qu'une cristallisation suspendue entre deux oralités, où il convient de chercher, aujourd'hui, les voix et les pratiques de ceux qui s'incarnaient par personnages sur la scène du jeu et de l'esprit.

Taku KuroIwA - University of Tohoku, 1-1-2chome katahira, Aoba-ku, Sendai 980-007 Japon

Xavier Leroux - Université du Sud-Toulon Var, UFR de Lettres et Sciences Humaines, EA 2649, avenue de l'Université, BP 20132, 83957 La Garde cedex

Darwin SMith - Laboratoire de médiévistique occidentale de Paris, UMR 8589, CNRS, Université Paris I Panthéon-Sorbonne, 7bis rue Guy Môquet, 94801 Villejuif

64. Voir dans ce même dossier l'article de G. PARussa, «Éditer les textes de théâtre en langue française : aperçu historique et nouvelles perspectives ». 


\section{De l'oral à l'oral : réflexions sur la transmission écrite des textes dramatiques au Moyen Âge}

Ce présent travail vise à analyser le processus d'enregistrement du texte dramatique à deux niveaux. Il consiste d'abord en la structuration mentale d'un discours syntaxiquement organisé, le formatage, à l'aide de diverses conventions d'écriture comme la versification. Il procède ensuite d'une notation ou d'une transcription à usage tant théâtral que non-théâtral, la formalisation, dont la logique de travail répond à chaque situation de réalisation matérielle, comme la performance théâtrale, la lecture, la méditation, l'enseignement, etc. Les témoins scriptuaires obtenus se laissent difficilement répartir dans les catégories utilisées jusqu'à présent.

La prise en compte de la formalisation et du formatage nous amène à éclairer les irrégularités métriques ou rimiques, insertions et autres procédés d'élaboration comme autant de témoins de la vie performative (ou active) de la matière transmise. L'historiographie du théâtre médiéval s'est longtemps attachée à distinguer les bons des mauvais manuscrits sans tenir compte de la mouvance textuelle inhérente à la transmission des textes. Les critères d'analyse que nous avons tenté d'établir permettent de réévaluer les différents représentants d'une famille en termes, non de qualité, mais de nature et de fonction, afin d'aboutir à une conception globalisante des mécanismes de production mentale et écrite des textes dramatiques.

manuscrit - théâtre - oral - écrit - transmission - enregistrement - versification - performance.

\section{From the oral to the oral : reflections on the written transmission of dramatic texts in the Middle Ages}

This work aims to analyze, at two levels, the process of recording dramatic texts. It initially examines the mental structuring of a syntactically organized speech, what may be called: the layout, which is achieved by means of various writing conventions, such as versification. It goes on to look at notation or transcription of theatrical and non-theatrical processes, which we call here: the formalization. This type of notation must respond to every situation of material realization, which may include, the theatrical performance, reading, meditation, teaching, and other activities around the text. The written examples or, more technically, the witnesses of these notation processes are difficult to ascribe to the current categories.

The consideration of formalization and layout exposes the metric irregularities or rhythmic patterns and the other processes of elaboration which highlight the performative or active life of the material text. The historiography of medie- 
val theatre has attempted for a long time to distinguish between good and bad manuscripts without taking into account the shifts or textual tendencies inherent in the transmission of texts. The criteria of analysis that we try to establish here permit a re-evaluation of the terminology, not from a standpoint of quality, but of nature and function, with the aim of arriving at an all-embracing conception of the mechanisms of the mental and written production of dramatic texts.

manuscript - theatre - oral - paper - transmission - recording - versification performance. 